\title{
Intoxicação por Solanum paniculatum (Solanaceae) em bovinos ${ }^{1}$
}

\author{
Eduardo Levi de Sousa Guaraná ${ }^{*}$, Franklin Riet-Correa ${ }^{3}$, Carla Lopes \\ de Mendonça ${ }^{4}$, Rosane M.T. Medeiros ${ }^{3}$, Nivaldo de Azevêdo Costa ${ }^{4}$ \\ e José Augusto Bastos Afonso ${ }^{4}$
}

\begin{abstract}
Guaraná E.L.S., Riet-Correa F., Mendonça, C.L., Medeiros R.M.T., Costa N.A. \& Afonso J.A.B. 2011. [Poisoning by Solanum paniculatum (Solanaceae) in cattle.] Intoxicação por Solanum paniculatum (Solanaceae) em bovinos. Pesquisa Veterinária Brasileira 31(1):59-64. Clínica de Bovinos, Campus Garanhuns, Universidade Federal Rural de Pernambuco, Av. Bom Pastor s/n, Cx. Postal 152, Mundaú, Garanhuns, PE 55292-901, Brazil. E-mail: eduardoguarana@ hotmail.com

Outbreaks of a disease of the nervous system are reported in cattle in three farms in the Agreste region of the state of Pernambuco. Morbidity, mortality and fatality rates varied from 3 to $25 \%, 0$ to $20 \%$ and 0 to $60 \%$, respectively. A weed found in large amounts in the pastures was identified as Solanum paniculatum. Clinical signs were characterized by transitory, periodic attacks with loss of balance, incoordinated gait, neck and head extension, hypermetria, intention tremors, nystagmus, and falls. The attacks were induced when the animals were disturbed or by the application of the head raising test. Two cows showed permanent signs including ataxia, abnormal posture, staggering gait with limbs in abduction, intention tremors, hypermetria, and progressive weight loss. Histological lesions in one cow were fine vacuolation of the cerebellar Purkinje neurons with marginalization of the nucleus. Loss of Purkinje neurons with proliferation of Bergmann astrocytes and Wallerian degeneration with axonal spheroids in the granular layer and cerebellar white matter were also observed. Neuronal vacuolation and axonal spheroids were observed in the gracillis nucleus. In one cow that stayed for approximately 10 months in an area free of $S$. paniculatum with permanent signs, there was a severe depletion of Purkinje neurons in the cerebellum. The granular and molecular layers were reduced and depleted of cells. Considering that the toxic compound of $S$. paniculatum is unknown, and that the plant is largely used as a medical plant, it is necessary to take into account the risk of human poisoning.
\end{abstract}

INDEX TERMS: Poisonous plants, Solanum paniculatum, Solanaceae, plant poisoning, central nervous system, storage disease, cerebellar degeneration, cattle.

RESUMO.- Surtos de uma doença neurológica com sinais cerebelares ocorreram em três fazendas da região Agreste do Estado de Pernambuco. A morbidade foi de 3 a $25 \%$,

\footnotetext{
${ }^{1}$ Recebido em 26 de julho de 2010.

Aceito para publicação em 11 de setembro de 2010.

2 Programa de Pós-Graduação em Ciência Veterinária, Universidade Federal Rural de Pernambuco (UFRPE), Rua Dom Manoel de Medeiros s/n, Dois Irmãos, Recife, PE 52171-900, Brasil. *Autor para correspondência: eduardoguarana@ hotmail.com

${ }^{3}$ Hospital Veterinário, Centro de Saúde e Tecnologia Rural (CSTR), Universidade Federal de Campina Grande, Campus de Patos, 58700000 Patos, PB, Brasil.

${ }^{4}$ Clínica de Bovinos, Campus Garanhuns, UFRPE, Av. Bom Pastor s/n, Cx. Postal 152, Garanhuns, PE 55292-901, Brasil.
}

a mortalidade variou de 0 a $20 \%$ e a letalidade foi de 0 a $60 \%$. Uma planta que predominava nos pastos das fazendas foi identificada como Solanum paniculatum. Os sinais clínicos apresentados foram de crises periódicas caracterizadas por incoordenação, extensão da cabeça e pescoço, ataxia, hipermetria, tremores de intenção, nistagmo e quedas. As crises eram induzidas pelo teste de levantar a cabeça ou quando os animais eram assustados ou quando aplicado o teste de levantar a cabeça. Alguns animais apresentaram sinais permanentes com alterações posturais, tremores de intenção, andar cambaleante com os membros em abdução e perda progressiva de peso. De dois bovinos que foram necropsiados, um apresentava di- 
minuição de tamanho do cerebelo com marcada atrofia da substância cinzenta. Histologicamente, um dos bovinos apresentou vacuolização fina do pericário das células de Purkinje do cerebelo com marginalização do núcleo. Em algumas áreas havia perda de neurônios de Purkinje com proliferação de astrócitos de Bergmann. Degeneração do tipo Walleriana, com esferoides axonais e vacúolos, alguns contendo macrófagos, foi observada na camada granular do cerebelo, substância branca cerebelar e medula cerebelar. Neurônios vacuolizados e esferóides axonais foram observados também no núcleo gracilis. Em outro bovino com sinais permanentes, que permaneceu por aproximadamente 10 meses sem ter acesso a S. paniculatum, observou-se ausência quase total de células de Purkinje. Havia severa depleção das camadas granular e molecular que se encontravam marcadamente diminuídas de espessura e com rarefação do neurópilo e menor número de células. Considerando que se desconhece o princípio ativo de $S$. paniculatum e que a planta é largamente utilizada como planta medicinal é necessário alertar para os riscos de intoxicação em humanos.

TERMOS DE INDEXAÇÃO: Plantas tóxicas, Solanum paniculatum, Solanaceae, intoxicação por planta, sistema nervoso central, doença de armazenamento, degeneração cerebelar, bovinos.

\section{INTRODUÇÃO}

Muitas doenças do armazenamento lisossomal de humanos e animais afetam o sistema nervoso central (SNC), com consequentes distúrbios neurológicos (Ralph 1990). Caracterizam-se por acúmulo intraneuronal de produtos metabólicos indigeríveis, que se acumulam nos lissosomos devido à atividade deficiente de uma, dentre diversas enzimas catabólicas lisossômicas, sendo classificadas como genéticas ou adquiridas. As adquiridas resultam da ingestão de plantas que contêm inibidores específicos de uma ou mais enzimas catabólicas lisossômicas. Plantas dos gêneros Astragalus, Oxytropis e Swainsona, conhecidas como "locoweed", causam $\alpha$-manosidoses em equinos, bovinos, ovinos e caprinos no oeste do Canadá, Estados Unidos, nordeste do México e Austrália (James et al. 1981, Smith 2006).

No Brasil, as doenças de depósito lisossomal induzidas pela ingestão de plantas incluem manosidoses causadas por Ipomoea carnea subsp. fistulosa (Antoniassi et al. 2007, Armien et al. 2007, Oliveira et al. 2009), I. sericophylla, I. riedelii (Barbosa et al. 2007), Turbina cordata (Dantas et al. 2007) e Sida carpinifolia (Driemeier et al. 2000), lipofuscinose causada por Phalaris angusta (Barros et al. 2006) e uma neurolipidose causada por Solanum fastigiatum var. fastigiatum (Riet Correa et al. 1983, Rech et al. 2006). S. fastigiatum var. fastigiatum é um arbusto que atinge até $1 \mathrm{~m}$ de altura, com folhas largas e flores brancas, sendo conhecida popularmente como "joá-preto" ou "jurubeba" (Riet-Correa et al. 1983, Barros et al. 1987). Desconhecese o princípio ativo da planta, que apresenta lesões semeIhantes às do grupo das neurolipidoses (Barros et al. 1987). É possível que tenha como principio tóxico algum inibidor enzimático ou substância que favoreça a formação de complexos lipídicos resistentes ao metabolismo causando uma doença neurológica caracterizada por ataques convulsivos transitórios (Barros et al. 1987). Algumas das alterações observadas na microscopia eletrônica se assemelham as observadas nas gangliosidoses (Riet-Correa et al. 1983). Os neurônios são ricos em gangliosídeos e glicolipídios, que são continuamente degradados e resintetizados em moléculas complexas. Distúrbios do metabolismo dos gangliosídeos resultam no acúmulo dos subprodutos degradativos nos neurônios, e em outras células, causando uma sobrecarga dos lisossomos pelo material indigesto e produzindo uma profunda disfunção neurológica (Smith 2006). Diversas espécies de Solanum causam doenças semeIhantes em diversos países: S. kwebense na África do Sul (Pienaar et al. 1976), S. dimidiatum nos EUA (Menzies et al. 1979) e S. bonariense no Uruguai (Verdes et al. 2006) afetam bovinos e S. cinereum na Australia (Bourke 1997) e S. viarium nos EUA (Porter et al. 2003) afetam caprinos.

O objetivo deste trabalho foi descrever, pela primeira vez, a intoxicação natural por Solanum paniculatum em bovinos. Para isso são relatados três surtos que foram diagnosticados na Clínica de Bovinos, Campus Garanhuns da Universidade Federal Rural de Pernambuco (UFRPE), na região do Agreste do Estado de Pernambuco.

\section{MATERIAL E MÉTODOS}

As informações foram resgatadas a partir de visitas realizadas em três propriedades localizadas nos municípios de Brejão, Poção e Pesqueira no Agreste do Estado de Pernambuco. Os dados epidemiológicos foram obtidos junto aos proprietários em visitas realizadas às propriedades. Uma amostra da planta de gênero Solanum suspeita de ser a causa da doença foi coletada e enviada para identificação botânica ao Setor de Botânica da Universidade Federal Rural de Pernambuco.

$\mathrm{Na}$ ocasião da visita às propriedades investigadas, os animais foram submetidos ao exame clínico seguindo as recomendações de Dirksen et al. (1993). Para verificar sinais cerebelares, foi aplicado o teste de levantar a cabeça (head raising test), descrito por Pienaar et al. (1976), que consiste em levantar a cabeça do animal durante um minuto e soltá-la logo após; se ocorrer uma crise com perda de equilíbrio e queda (crise epileptiforme) o teste é considerado positivo. Paralelamente, foram coletadas amostras de sangue de seis bovinos, em tubo a vácuo com anticoagulante EDTA (10\%), para realização do hemograma, determinação da proteína plasmática total e do fibrinogênio plasmático, seguindo as recomendações de Jain (1986).

Duas vacas afetadas foram necropsiadas: uma que apresentava evolução de seis meses e ficou em decúbito permanente foi eutanasiada e necropsiada na propriedade; a outra após 10 meses de internamento na Clínica de Bovinos foi , também, eutanasiada devido ao agravamento da condição clínica. Para análise histopatológica, coletaram-se fragmentos de órgãos das cavidades abdominal e torácica, que foram fixados em solução tamponada de formol a 10\%, incluídos em parafina, cortados a 4-5 $\mu \mathrm{m}$, e corados pela Hematoxilina e Eosina (HE). O encéfalo e a medula foram fixados na íntegra; posteriormente foram realizados cortes transversais abrangendo amostras do córtex cerebral, cápsula interna, tálamo, tubérculos quadrigêmeos, gânglios paravertebrais, cerebelo, ponte, bulbo e medu- 
la espinhal cervical, dorsal e lombar que foram incluídas em parafina, cortados a 4-5 $\mu \mathrm{m}$ e coradas por HE.

\section{RESULTADOS}

\section{Epidemiologia.}

Os surtos ocorreram durante um período de três anos, entre os meses de setembro 2005 e dezembro 2008, em bovinos utilizados para produção de leite das raças Holandês, Pardo-Suiço e seus cruzamentos, com idades de dois a cinco anos. Em todos os surtos os animais permaneciam em pastos invadidos por uma planta do gênero Solanum, que era denominada vulgarmente de "jurubeba", identificada como Solanum paniculatum L. (Fig.1) e registrada no Herbário da Universidade Federal Rural de Pernambuco (PEUFR) com no 50.434. Os índices de morbidade, mortalidade e letalidade das propriedades onde ocorrem os surtos podem ser observados no Quadro 1. Uma característica observada em duas propriedades foi que os pastos eram roçados, com isso a brotação de S. paniculatum encontrava-se entre as pastagens, o que provavelmente facilitava a sua ingestão (Fig.1C).

\section{Sinais clínicos}

Os sinais neurológicos se caracterizaram por crises periódicas, epileptiformes, com quedas, que surgiam geralmente quando os animais eram assustados ou forçados a correr. Durante as crises os animais apresentavam nistagmo, opistótono, extensão dos membros anteriores e posteriores, tremores e quedas (Fig.2). Após alguns segundos ou minutos se levantavam, alguns com dificulda-
Quadro 1. Morbidade, mortalidade e letalidade em três propriedades onde ocorrem surtos de intoxicação por Solanum paniculatum

\begin{tabular}{lcccc}
\hline Propriedade & 1 & 2 & 3 & Total \\
\hline Município & Brejão & Poção & Pesqueira & \\
Animais doentes & 15 & 7 & 3 & 25 \\
Animais mortos & 12 & 3 & 0 & 15 \\
Efetivo do rebanho & 60 & 120 & 100 & 280 \\
Morbidade (\%) & 25 & 5,83 & 3 & 8,9 \\
Mortalidade (\%) & 20 & 2,5 & 0 & 5,3 \\
Letalidade (\%) & 80 & 42,85 & 0 & 60
\end{tabular}

de, e retornavam ao normal, sem apresentar sinais entre uma crise e outra. As crises se repetiam com periodicidade variável, mas podiam ser induzidas se os animais eram assustados ou movimentados e após a aplicação do teste de levantar a cabeça. Os bovinos acometidos em sua maioria apresentavam escore corporal regular apesar de poucos apresentarem emagrecimento progressivo e apetite normal. Os parâmetros cardíacos, respiratórios e ruminais se encontravam normais. Dois animais apresentaram sinais permanentes: um foi eutanasiado na fazenda por estar em decúbito permanente e o outro permaneceu na Clínica de Bovinos de Garanhuns por 10 meses. Este último apresentava ataxia, hipermetria, hiperestesia, tremores de intenção, posturas anormais, andar cambaleante lento, extensão dos membros (principalmente os anteriores), lateralização do andar e quando estimulado caia demonstrando crises epileptiformes com opistótono e nistagmo. Durante os 10 meses permaneceu com o quadro neurológico estável, mesmo sem estar em contato com a
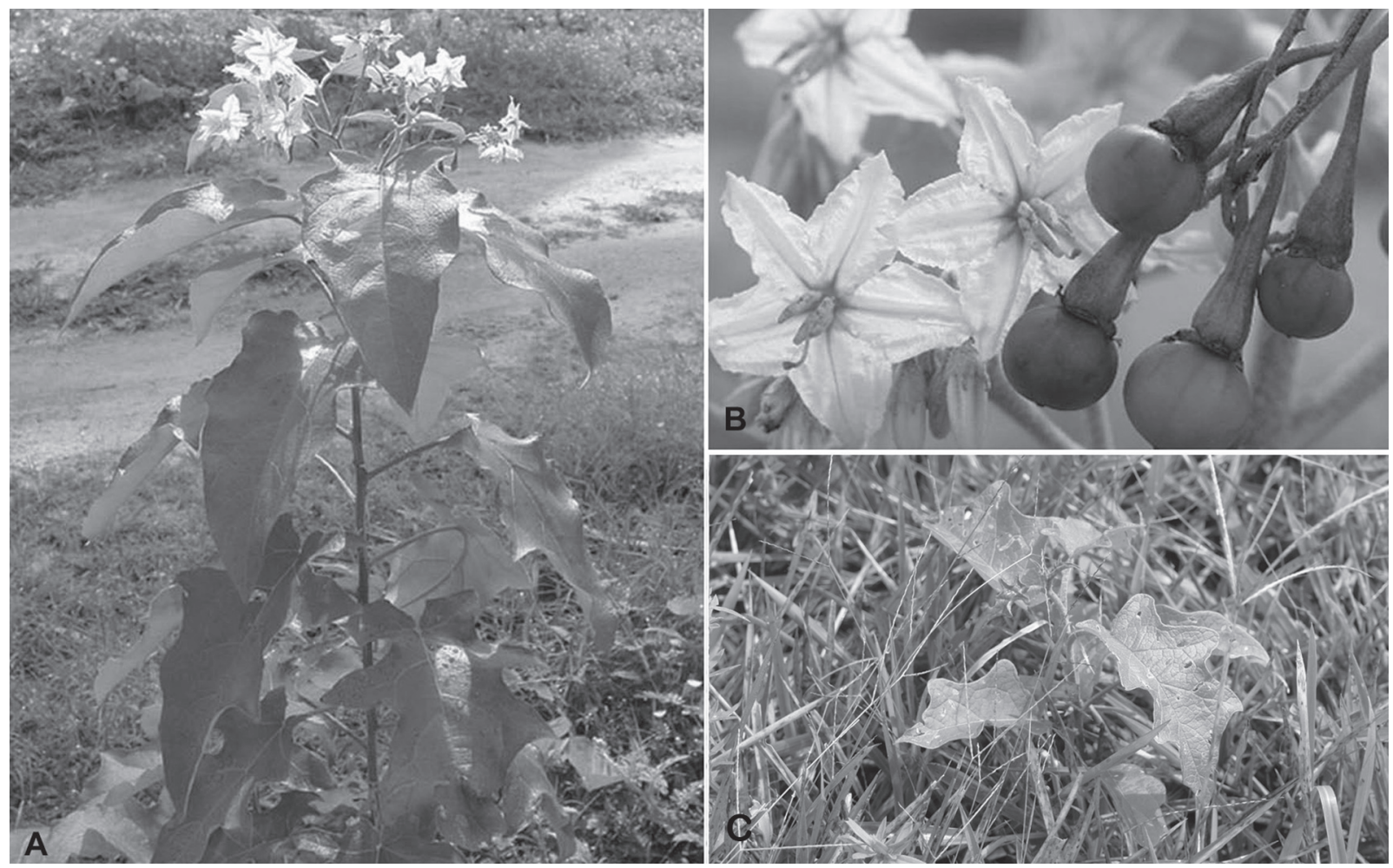

Fig.1. Solanum paniculatum. (A) Planta. (B) Flores e frutos. (C) Brotação misturada com a pastagem. 


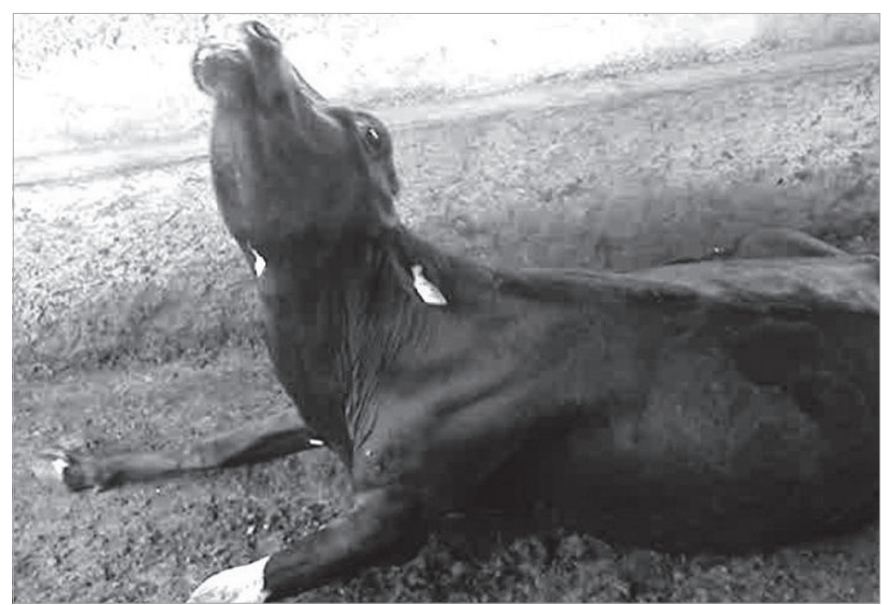

Fig.2. Animal intoxicado espontaneamente apresentando crise neurológica após o teste de levantar a cabeça.

planta. Animais com crises periódicas, sem acesso a planta, mantiveram quadro clínico estável, ganharam peso e demonstraram sinais apenas quando bastante estimulados ou estressados.

Com o objetivo de evitar o aparecimento de novos casos de intoxicação ou perda com morte de animais, os proprietários foram orientados que retirassem os bovinos do pasto que possuía jurubeba, e que colocassem os animais que já apresentavam sintomatologia nervosa em local de fácil manejo e sem estresse. As mortes estavam relacionadas a acidentes (afogamento ou traumatismo) ocorridos durante uma crise e devido à prostração com a dificuldade de levantar.

Não foram observadas alterações nos valores do hemograma, bem como da concentração plasmática da proteína total e do fibrinogênio (Kramer 2000).

Na necropsia a única alteração significante foi a diminuição de tamanho do cerebelo do Bovino 2, que apresentava marcada atrofia da substância cinzenta (Fig.3A) No Bovino 1 não foram encontradas lesões macroscópicas de significação.

Histologicamente, no Bovino 1, a maioria dos neurônios de Purkinje apresentavam vacuolização fina do pericário, com marginalização do núcleo (Fig.3B). Em algumas áreas havia perda de neurônios de Purkinje com proliferação de astrócitos de Bergmann. Esferoides axonais eram observados na camada granular do cerebelo (Fig.3B), na substância branca cerebelar e na medula cerebelar, principalmente nesta última. Vacuolos (elipsóides de mielina), alguns contendo macrófagos no seu interior, eram também observados. Neurônios vacuolizados e esferóides axonais foram observados também no núcleo gracilis (Fig.3C). No Bovino 2, que permaneceu por aproximadamente 10 meses na Clínica de Bovinos de Garanhuns, sem ter acesso a $S$. paniculatum, observava-se ausência quase total de células de Purkinje; as raras células de Purkinje remanescentes apresentavam vacuolização fina do pericario e núcleo marginalizado. Havia severa depleção das camadas granulare molecular (Fig.3D que se encontravam marcadamente diminuídas de espessura e com rarefação do neurópilo e menor número de células quando comparadas com cerebelos de animais normais ou com o cerebelo do Bovino 1. Raros esferóides axonais eram observados na camada granular, sustância branca cerebelar e medula cerebelar.

\section{DISCUSSÃO}

O diagnóstico da intoxicação por Solanum paniculatum foi baseado nos sinais clínicos observados, na presença da planta e pela observação das alterações histológicas características, relatados em intoxicações por outras espécies de Solanum em bovinos (Pienaar et al. 1976, Menzies et al. 1979, Riet-Correa et al. 1983, Rech et al. 2006, Verdes et al. 2006) e reproduzidas experimentalmente com $S$. paniculatum (Barros et al. 1987, Medeiros et al. 2004, Guaraná et al. 2009). Alem das lesões histológicas a atrofia das camadas molecular e granular do cerebelo e a marcada ausência de células de Purkinje foram descritas anteriormente nas intoxicações por S. fastigiatum var. fastigiatum (Rech et al. 2006) e S. kwebense (Van der Lugt et al. 2010).

S. paniculatum é uma invasora de pastagens ou de terrenos abandonados muito frequente nas regiões Norte e Nordeste, mas encontrada em todo o País. Barros et al. (1987), no Rio Grande do Sul, testaram experimentalmente a toxicidade de $S$. fastigiatum var. fastigiatum e de outra Solanacea, Solanum sp., posteriormente identificado como S. paniculatum. Esta última causou sinais clínicos, idênticos aos causados por $S$. fastigiatum var. fastigiatum, após a administração da planta verde ad libitum durante 639 dias ou da planta seca por fistula ruminal durante 142 dias; os primeiros sinais foram observados após 260 dias com a planta verde e após 160 dias com a planta seca. Em um experimento, com planta coletada na Paraíba, Medeiros et al. (2004) observaram sinais clínicos após a administração da planta seca, via fístula ruminal, na dose de $5 \mathrm{~g} / \mathrm{kg} /$ dia durante 93 a 142 dias. Em outro experimento com planta coletada no Estado de Pernambuco foram reproduzidos sinais clínicos e lesões características após a administração de $3 \mathrm{~g} / \mathrm{kg}$ de planta seca por $\mathrm{kg}$ de peso durante 3 meses e $4 \mathrm{~g} / \mathrm{kg}$ durante 30 dias; os primeiros sinais foram observados após 120 dias de consumo da planta (Guaraná et al. 2009). Por esses resultados é evidente que são necessárias grandes quantidades da planta para induzir sinais clínicos, o que sugere que a intoxicação espontânea ocorre, principalmente, quando a mesma se encontra rebrotando entre as pastagens, como aconteceu nas fazendas onde ocorreram os surtos. Outro fator determinante da aparentemente baixa frequência da intoxicação no Nordeste, apesar da ampla distribuição da planta, é a sua pouca palatabilidade, que faz com que na maioria das ocasiões os animais não a consumam mesmo em condições de escassez de forragem. Além disso, a planta cresce durante o período de chuvas, quando geralmente há outras forragens disponíveis.

Nos animais com quadro clínico não muito severo, que deixaram de ingerir a planta, os sinais se tornaram mais brandos e esporádicos, sugerindo que algum grau de reversão das lesões nesta situação possa ocorrer. Entretan- 

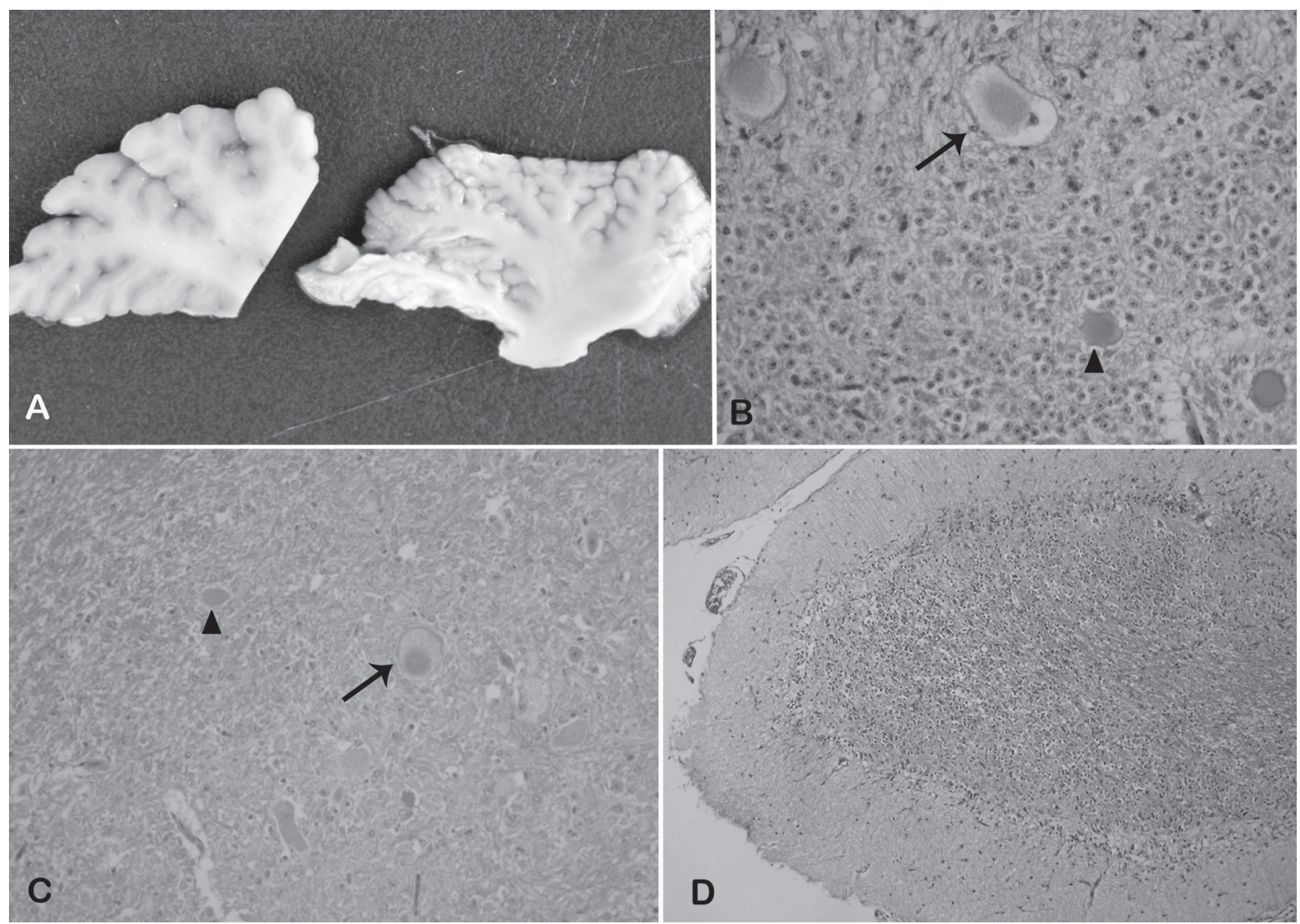

Fig.3. (A) Cerebelo do Bovino 2 (à direita) e de um bovino controle (esquerda). Observa-se severa atrofia do córtex cerebelar no Bovino 2. (B) Cerebelo do Bovino 1. Observa-se a severa vacuolização de neurônios de Purkinje com núcleos marginalizados e picnóticos (seta) e esferóides axonais na camada granular (cabeça de seta). HE, obj.20x. (C) Núcleo gracilis do Bovino 1. Observa-se neurônio severamente vacuolizado (seta) e esferoides axonais (cabeça de seta). HE, obj.20x. (D). Cerebelo do Bovino 2. Observa-se ausência de neurônios de Purkinje, proliferação da glia de Bergman e marcado estreitamento e rarefação das camadas molecular e granular.

to, nos animais com sinais mais severos (crises mais frequentes) ou naqueles com sinais permanentes, não houve melhora. Zambrano et al. (1985) sugerem que as lesões do pericario (vacuolização) e axônios (esferóides axonais) das células de Purkinje são lentamente reversíveis. Após a morte celular a lesão não é mais reversível e há um processo de degeneração tipo Walleriana. A severa depleção observada nas camadas granular e molecular do cerebelo são, aparentemente, consequência da remoção de axônios e dendritos das células de Purkinje.

Para as medidas preventivas é necessário evitar as condições epidemiológicas mencionadas acima ou, no caso de que estas ocorram, limitar o período de permanência dos animais em pastagens muito invadidas. Por outro lado devemos levar em consideração os prováveis riscos de intoxicação em humanos, já que $S$. paniculatum é largamente utilizada com planta medicinal, para preparação de bebidas (vinho de jurubeba) ou como alimento (conserva) (Santos Neto et al. 2006).

Análises nas raízes de Solanum spp. mostram uma multiplicidade de alcalóides ou a presença de compostos que representem novos tipos estruturais. Umm novo alcalóide esteróide, paniculidina, e seu glicosídeo, paniculina foram identificados em raízes de $S$. paniculatum. Em raízes de plantas cultivadas na Europa foi identificado um glicosídeo, juribina, que por hidrólise enzimática ou ácida, produz $1 \mathrm{~mol}$ D-glicose e uma alcamina (jurubidina), representando este último composto um alcalóide esteróide de novo tipo estrutural (Schreiber 1968), no entanto, não tem sido demonstrada se alguma dessas substâncias causa o quadro clínico.

Agradecimentos.- À Professora Dra. Suzene Izídio da Silva do Departamento de Biologia, Área de Botânica/UFRPE, pela identificação da planta local. Ao Instituto Nacional de Ciência e Tecnologia (INCT) para o Controle das Intoxicações por Plantas (MCT, CNPq), pelo suporte financeiro (Proc.573534/2008-0).

\section{REFERÊNCIAS}

Antoniassi N.A.B., Ferreira E.V., Santos C.E.P., Campos J.L.E., Nakazato L. \& Colodel E.M. 2007. Intoxicação espontânea por Ipomoea carnea subsp. fistulosa (Convolvulaceae) em bovinos no Pantanal Matogrossense. Pesq. Vet. Bras. 27(10):415-418.

Armién A.G., Tokarnia C.H., Peixoto P.V. \& Frees K. 2007. Spontaneous and experimental glycoprotein storage disease of goats induced by Ipomoea carnea subsp fistulosa (Convolvulaceae). Vet. Pathol. 44:170-184. 
Barbosa R.C., Riet-Correa F., Lima E.F., Medeiros R.M.T., Guedes K.M.R, Gardner D.R., Molyneux R.J. \& Melo L.E.H. 2007. Experimental swainsonine poisoning in goats ingesting Ipomoea sericophylla and Ipomoea riedelii (Convolvulaceae). Pesq. Vet. Bras. 27(10):409-414.

Barros S.S., Riet-Correa F., Andujar M.B., Barros C.S.L., Méndez M.C. \& Schild A.L. 1987. Solanum fastigiatum and Solanum sp poisoning in cattle: Ultraestructural changes in the cerebellum. Pesq. Vet. Bras. 7:1-5.

Barros C.S.L., Driemeier D., Dutra I.S. \& Lemos R.A.A. 2006. Doenças do Sistema Nervoso de Bovinos no Brasil. Coleção Vallée, São Paulo, p.110-112.

Bourke C.A. 1997. Cerebellar degeneration in goats grazing Solanum cinereum (Narrawa burr). Aust. Vet. J. 75:363-365.

Dantas A.F.M., Riet-Correa F., Gardner D.R., Medeiros R.M.T., Barros S.S., Anjos B.L. \& Lucena R.B. 2007. Swainsonine-induced lysosomal storage disease in goats caused by the ingestion of Turbina cordata in Northeastern Brazil. Toxicon 49:111-116.

Dirksen G., Gründer H.D. \& Stöber M. 1993. Rosenberger, Exame Clínico dos Bovinos. $3^{a}$ ed. Guanabara Koogan, Rio de Janeiro. 419p.

Driemeier D., Colodel E.M., Gimeno E.J. \& Barros S.S. 2000. Lysosomal storage disease caused by Sida carpinifolia poisoning in goats. Vet. Pathol. 37:153-159.

Guaraná E.L.S., Riet-Correa F., Mendonça C.L., Medeiros M.T., Costa N.A. \& Afonso J.A.B. 2009. Poisoning by Solanum paniculatum in cattle in Pernambuco, Northeastern Brazil. Proceedings $8^{\text {th }}$ International Symposium on Poisonous Plants, João Pessoa, Brazil, p.62.

Jain N.C. 1986. Schalm's Veterinary Hematology. $4^{\text {th }}$ ed. Lea and Febinger, Philadelphia. 1221p.

James L.F., Hartley W.J. \& Van-Kampen K.R. 1981. Syndromes of Astragalus poisoning in livestock. J. Am. Vet. Med. Assoc. 178:146-150.

Kramer J.W. 2000. Normal hematology of cattle, sheep and goats, p.1075-1084. In: Feldman B.F., Zinkl J.G. \& Jain N.C. (Eds), Schalm's Veterinary Hematology. $5^{\text {th }}$ ed. Williams and Wilkins, Philadelphia.

Medeiros R.M.T., Guilherme R.F., Riet-Correa F., Barbosa R.C. \& Lima E.F. 2004. Experimental poisoning by Solanum paniculatum (jurubeba) in cattle. Pesq. Vet. Bras. 24(Supl.):41.

Menzies J.S., Bridges C.H. \& Bailey E.M. 1979. A neurological disease of cattle associated with Solanum dimidiatum. South Western Vet. 32:45-49.

Oliveira C.A., Barbosa J.D., Duarte M.D., Cerqueira V.D., Riet-Correa F. \& Riet-Correa G. 2009. Intoxicação por Ipomoea carnea subsp. fistulosa (Convolvulaceae) em caprinos na Ilha do Marajó, Pará. Pesq. Vet. Bras. 29(7):583-588.

Pienaar J.G., Kellerman T.S., Basson P.A., Jenkins W.L. \& Vahrmeijer J. 1976. Maldronksiekte in cattle: A neuronopathy caused by Solanum kwebense. Onderstepoort J. Vet. Res. 43:67-74.

Porter M.B., Mac Kay R.J., Uhl E., Platt S.R \& de Lahunta A. 2003. Neurologic diseases putatively associated with Solanum viarium in goats. J. Am. Vet. Med. Ass. 223(4):501-504.

Ralph W.S. 1990. Sistema Nervoso Central, p.579-643. In: Thomson R.G. (Ed.), Patologia Veterinária Especial. Manole, São Paulo.

Rech R.R., Rissi D.R., Rodrigues A., Pierezan F., Piazer J.V.M., Kommers G.D. \& Barros C.S.L. 2006. Intoxicação por Solanum fastigiatum (Solanaceae) em bovinos: epidemiologia, sinais clínicos e morfometria das lesões cerebelares. Pesq. Vet. Bras. 26:183-189.

Riet-Correa F., Méndez M.C., Schild A.L., Summers B.A. \& Oliveira J. A. 1983. Intoxication by Solanum fastigiatum var. fastigiatum as a cause of cerebellar degeneration of cattle. Cornell Vet. 73:240-256.

Santos Neto O.D., Karsburg I.V. \& Yoshitome M.Y. 2006. Viabilidade e germinabilidade polínica de populações de jurubeba (Solanum paniculatum I.). Revta Ciênc. Agro-Ambientais, Alta Floresta 4(1):67-74.

Schreiber K. 1968. Steroid Alkaloids: The Solanum group, p.1-82. In: Manske R.H.F. (Ed.), The Alkaloids: Chemistry and pharmacology. Vol.10. Academic Press, New York.

Smith M.O. 2006. Doenças do Sistema Nervoso, p.872-1018. In: Smith B.P. (Ed.), Tratado de Medicina Interna de Grandes Animais. Manole, São Paulo.

Van der Lugt J.J., Bastianello S.S., Van Ederen A.N. \& Van Wilpe E. 2010. Cerebellar cortical degeneration in cattle caused by Solanum kwebense. Veterinary Journal 185(2):225-227.

Verdes J.M., Moraña A., Gutierrez F. Goicoa A., Fidalgo L.E. \& Guerrero F. 2004. Cerebellar deeneration in cattle grazing Solanum bonariense (naranjillo) in Western Uruguay. J. Vet. Diag. Invest. 18(3):299-303.

Zambrano M., Riet-Correa F., Schild A.L. \& Méndez M.C. 1985. Intoxicação por Solanum fastigiatum var. fastigiatum. Pesq. Vet. Bras. 5(4):133-141. 